\title{
Effects of Photoluminescence Polarization in Semiconductor Quantum Well Subjected to In-Plane Magnetic Field
}

\author{
Yu.G. SEMENOV \\ Institute of Semiconductor Physics, NAS of Ukraine \\ 45, Prospekt Nauki, Kiev 03028, Ukraine \\ AND S.M. RYABCHENKO \\ Institute of Physics, NAS of Ukraine \\ 46, Prospekt Nauki, Kiev 03028, Ukraine
}

\begin{abstract}
Strong optical polarization anisotropy observed previously in the exciton photoluminescence from [100]-oriented quantum wells subjected to the in-plane magnetic field is described within microscopic approach. Developed theory involves two sources of optical polarization anisotropy. The first of them is due to correlation between $\psi$-functions phases of electron and heavy hole which arise owing to electron Zeeman spin splitting and joint manifestation of low-symmetrical and Zeeman interactions of heavy holes in an in-plane magnetic field. Other optical polarization anisotropy source stems from the admixture of light-holes states to heavy-holes ones by low-symmetry interactions. The heavy hole splitting caused by these interactions separately and the effects of their interference are analyzed. The domination of $C_{2 v}$ low-symmetry interaction connected with quantum wells interfaces and/or in-plane deformations takes place in relatively low magnetic field. The directions of this perturbation determine main directions of the $\pi$-periodical optical polarization anisotropy. The cubic anisotropy of valence band can add the $\pi / 2$-periodical contribution to the optical polarization anisotropy. In the case of quantum wells with semimagnetic barriers the Zeeman term contribution can reach value, which dominates the $C_{2 v}$ ones, and crossover to polarization connected with magnetic field direction may be observed in low temperature.
\end{abstract}

PACS numbers: 78.67.-n, 78.20.Ls 


\section{Introduction}

The linear polarization $\rho$ of photoluminescence (PL) in quantum wells (QWs) is sensitive to relatively weak low symmetry interactions $V$, which mix the light hole $(\mathrm{LH})$ and heavy hole $(\mathrm{HH})$ states $[1-5]$. One could expect due to this virtue the polarization magnitude about $\varepsilon=|V| / \Delta_{\mathrm{HL}} \ll 1$ ( $\Delta_{\mathrm{HL}}$ is HH-LH energy splitting). In contrast with such estimation of $\varepsilon$ a strong polarization of PL from [001]-oriented QW $\mathrm{Cd}_{1-x} \mathrm{Mn}_{x} \mathrm{Te} / \mathrm{CdTe} / \mathrm{Cd}_{1-x} \mathrm{Mn}_{x} \mathrm{Te}$ and its anisotropy (i.e. dependence $\rho$ on sample rotation about QW normal) has been observed in Refs. [2, 4]. The polarization increased sharply with an increase in the in-plane magnetic field $\boldsymbol{B}$ and reaches a few tens of percents. The pseudospin formalism and the phenomenological describing of effect were developed in Ref. [2]. The microscopic approach taking into account the different interactions causing the $\mathrm{HH}$ splitting was used in $[4,5]$.

It is known that any interaction splitting the degenerate electron and $\mathrm{HH}$ levels imposes phase correlation between the electron and hole wave functions. This correlation forms the polarization and its anisotropy for electron-hole optical transitions between the pairs of distinguishable levels regardless of value of spin levels splitting. The small optical polarization anisotropy (OPA) caused by $\mathrm{LH}-\mathrm{HH}$ mixing is an additional one to this main contribution to OPA. The different interactions, which are able to lift the HH degeneracy, impose their specific correlation between electron and hole $\psi$-functions phases as well as the period and phase OPA. The effects of electron-hole exchange interaction inside the exciton are sensitive to the small $C_{2 v}$ perturbation, too [6]. Nevertheless, this interaction is small enough and, as it was discussed in Ref. [5], the effects connected with it may be neglected in most cases at the analysis of excitonic PL of the semimagnetic structures.

In this paper we summarize a performed in $[4,5]$ analysis of OPA caused by different low-symmetry interactions of a hole in [001]-oriented QW. It will be shown that they reveal the various OPA dependencies on in-plane magnetic field $\boldsymbol{B}$ rotation and qualitatively new peculiarities OPA arise due to interference effect of these contributions.

\section{Theoretical background}

Let us introduce the reference frame associated with structure axes so that $O Z$ is parallel to growth axis [001], while $O X \|[100]$ and $O Y \|[010]$ lie in QW plane. An in-plane magnetic field is directed at the angle $\varphi$ to $O X, \boldsymbol{B}=$ $B\{\cos \varphi, \sin \varphi, 0\}$. The polarization ratio of $\mathrm{PL}$ line in relation to polarization plane rotated relative to the $\boldsymbol{B}$ by angle about the $O Z$ axis (see Fig. 1) is

$$
\rho_{\alpha}=\left(I_{\alpha}-I_{\alpha^{\prime}}\right)\left(I_{\alpha}+I_{\alpha^{\prime}}\right)^{-1} \text {. }
$$

Here $I_{\alpha}$ is the integral intensity of $\mathrm{PL}$ with polarization plane $\alpha$; plane $\alpha^{\prime}$ is perpendicular to that $\alpha$. 
The 1e-1HH PL-spectrum involves four optical transitions from two electron spin sublevels to two HH ones. The electron (or HH) spin splitting $\omega=\omega_{\mathrm{e}}$ (or $\omega=\omega_{\mathrm{h}}$ ) is assumed to be described by following matrix Hamiltonian in certain basis $|n\rangle, n=1,2$ :

$$
\left\|H_{n, n^{\prime}}\right\|=\frac{\omega}{2}\left(\begin{array}{cc}
0 & \mathrm{e}^{-\mathrm{i} \theta} \\
\mathrm{e}^{\mathrm{i} \theta} & 0
\end{array}\right)
$$

where $\omega=2\left|H_{1,2}\right|, \sin \theta=-2 \operatorname{Im} H_{1,2} / \omega$. Eigenvalues and eigenfunctions of Hamiltonian (2) are

$$
E_{ \pm}= \pm \frac{\omega}{2}, \quad \psi^{ \pm}=\frac{1}{\sqrt{2}}\left( \pm \mathrm{e}^{-\mathrm{i} \theta / 2}|1\rangle+\mathrm{e}^{\mathrm{i} \theta / 2}|2\rangle\right)
$$

For the $c$-band electron, Hamiltonian $H_{e}=\boldsymbol{G}_{\mathrm{e}} \boldsymbol{s}$ takes the form (2) with $\theta=\varphi$ and $\omega_{e}=G_{e}$ in representation of $|1\rangle=S \uparrow$ and $|2\rangle=S \downarrow$. Here $\uparrow$ and $\downarrow$ are the eigenstates of spin projection $s_{z}, S$ is a periodic part of $c$-band Bloch function and $\boldsymbol{G}_{e} \| \boldsymbol{B}$ is an effective in-plane magnetic field (in energy units) that can include the effects of carrier-ion exchange interaction in the diluted magnetic semiconductor based (DMS-based) structures [7]. In the HH case, the basis $|1\rangle=L_{+} \uparrow$, $|2\rangle=-L_{-} \downarrow$ corresponds to $\pm 3 / 2$ projection of $\mathrm{HH}$ angular momentum on $O Z, L_{ \pm}=\frac{1}{\sqrt{2}}(X \pm \mathrm{i} Y), X$ and $Y$ are the periodic parts of $v$-band Bloch functions. The dependence $\theta(\varphi)$ has to be found for each specific form of HH Hamiltonian. the form

The operator of interband optical transition with polarization plane $\alpha$ takes

$$
\widehat{V}_{\alpha}=p_{-} \mathrm{e}^{\mathrm{i}(\varphi+\alpha)}+p_{+} \mathrm{e}^{-\mathrm{i}(\varphi+\alpha)},
$$

where $p_{ \pm}=\frac{1}{2}\left(e_{x} \pm \mathrm{i} e_{y}\right), e_{x}$ and $e_{y}$ are transformed as $x$ and $y$. The probabilities of optical transitions, $W_{k, j}^{\alpha}=\left\|M_{k, j}^{\alpha}\right\|^{2}=\left|\left\langle\psi_{c}^{k}\left|\widehat{V}_{\alpha}\right| \psi_{\nu}^{j}\right\rangle\right|^{2}$, between electron states $\psi_{c}^{k}, k= \pm 1$, and HH ones $\psi_{\nu}^{j}, j= \pm 1$ are

$$
W_{k, j}^{\alpha} \propto\left\{\begin{array}{cc}
\sin ^{2}(3 \varphi / 2+\alpha-\theta / 2), & k=j \\
\cos ^{2}(3 \varphi / 2+\alpha-\theta / 2), & k \neq j
\end{array}\right.
$$

Thus, Eq. (1) takes the form

$$
\rho_{\alpha}^{(0)}=\left[\sum_{k, j} P_{\mathrm{e}}^{k} P_{\mathrm{h}}^{j}\left(W_{k, j}^{\alpha}-W_{k, j}^{\alpha^{\prime}}\right)\right]\left[\sum_{k, j} P_{\mathrm{e}}^{k} P_{\mathrm{h}}^{j}\left(W_{k, j}^{\alpha}+W_{k, j}^{\alpha^{\prime}}\right)\right]^{-1} .
$$

where $P_{\mathrm{e}}^{k} \propto \mathrm{e}^{-k \omega_{\mathrm{e}} / 2 T_{\mathrm{e}}} /\left(\mathrm{e}^{\omega_{\mathrm{e}} / 2 T_{\mathrm{e}}}+\mathrm{e}^{-\omega_{\mathrm{e}} / 2 T_{\mathrm{e}}}\right)$ and $P_{\mathrm{h}}^{j} \propto \mathrm{e}^{-j \omega_{\mathrm{h}} / 2 T_{\mathrm{h}}} /\left(\mathrm{e}^{\omega_{\mathrm{h}} / 2 T_{\mathbf{h}}}\right.$ $\left.+e^{-\omega_{h} / 2 T_{h}}\right)$ are sublevel of electron and HH populations. Here $T_{\mathrm{e}}$ and $T_{\mathrm{h}}$ are the electron and HН spin temperatures (in energy units).

Substitution of (5) and expressions for $P_{\mathrm{e}}^{k}$ and $P_{\mathrm{h}}^{j}$ into (6) leads to the following simple result:

$$
\begin{aligned}
& \rho_{\alpha}^{(0)}=-P_{\mathrm{eh}} \cos (3 \varphi+2 \alpha-\theta), \\
& \left.P_{\mathrm{eh}}=\tanh \left(\omega_{\mathrm{e}} / 2 T_{\mathrm{e}}\right) 2 T_{\mathrm{e}}\right) \tanh \left(\omega_{\mathrm{h}} / 2 T_{\mathrm{h}}\right) .
\end{aligned}
$$


Equation (7) does not describe all possible polarization effects in QW. The low symmetry perturbations $V$ of HH basis wave functions $|1\rangle$ and $|2\rangle$ should also be taken into account along with $H H$ splitting in spite of small value $\varepsilon=$ $|\langle m|V| m \pm \Delta m\rangle| / \Delta_{\mathrm{HL}}$. Here $|m\rangle$ and $|m \mp \Delta m\rangle$ are non-perturbed HH $(|m|=3 / 2)$ and $\mathrm{LH}(|m \mp \Delta m|=1 / 2)$ basis functions. It gives rise to temperature independent corrections $\delta \rho_{\alpha} \sim \varepsilon^{3-\Delta m}$ to total polarization ratio $\rho_{\alpha}$ that can be now written as a sum

$$
\rho_{\alpha}=\rho_{\alpha}^{(0)}+\delta \rho_{\alpha} .
$$

Explicit form of $\delta \rho_{\alpha}$ depends on specific form of interaction $V$. Generally, the electron-HH optical line splits up into the four components with intensities $I_{\alpha k, j} \propto W_{k, j}^{\alpha} P_{\mathrm{e}}^{k} P_{\mathrm{h}}^{j}$. Each of them has a strong anisotropic polarization, $\rho_{\alpha k, j}^{(0)}=\left(W_{k, j}^{\alpha}-W_{k, j}^{\alpha^{\prime}}\right) /\left(W_{k, j}^{\alpha}+W_{k, j}^{\alpha^{\prime}}\right)=-k j \cos (3 \varphi+2 \alpha-\theta)$.

If the splitting of spectral components are smaller than their line widths $\sigma_{k j}$, the polarization $\rho$ depends on spectral position at the PL line contour $\omega$. The $\rho_{\alpha}(\omega)$ is defined by (1) with substitution $I_{\alpha}(\omega)=\sum_{k, j} I_{\alpha k, j}(\omega)$ instead of $I_{\alpha}$. The $I_{\alpha k, j}(\omega)$ depends on line shape of transitions $f_{k, j}(\omega)=f\left[\omega-\left(\omega_{0}+k \omega_{e} / 2-\right.\right.$ $\left.j \omega_{\mathrm{h}} / 2\right)$ ], where $\omega_{0}$ is the spectral position of the centre of gravity of split spectral quadruplet. The latter feels some shift in the in-plane magnetic field due to LH-HH mixing. The analysis of $\rho_{\alpha}(\omega)$ was performed in [5] for the cases of Gaussian and Lorentzian line shape and $\mathrm{HH}$ splitting smaller than $\sigma_{k j}$.

The wave functions phase correlation due to $\mathrm{HH}$ and electron spin splitting determines the OPA in the cases of absorption or reflectivity optical spectra, too. As distinct from PL, in these cases optical transitions occur between completely populated valence and empty conductivity electron states and the $\operatorname{limit} T_{\mathrm{e}}$ and $T_{\mathrm{h}} \rightarrow \infty$ should be used.

\section{The $\mathbf{H H}$ interactions}

Let us consider sequentially the HH interactions that lower the QW potential symmetry. And let us assume that they are small perturbations with respect to $\Delta_{\mathrm{HL}}$. In general, the effective Zeeman interaction in the semimagnetic structures can lead to the hole state splitting comparable with $\Delta_{\mathrm{HL}}$. The OPA in such case is connected with in-plane magnetic field direction. We will not analyze this case in detail here.

\subsection{Zeeman interaction}

Zeeman interaction $\mathrm{HH}$ with in-plane magnetic field is isotropic. In terms of hole effective angular momentum $J=3 / 2$ it has the form

$$
V_{Z}=\boldsymbol{G}_{\mathrm{h}} \boldsymbol{J}=G_{\mathrm{h}}\left(J_{X} \cos \varphi+J_{Y} \sin \varphi\right) .
$$

Here $\boldsymbol{G}_{\mathrm{h}} \| \boldsymbol{B}$ is an effective in-plane magnetic field for holes. This $V_{Z}$ does not split the $\mathbf{H H}$ states in first and second orders of perturbation. Third order can be 
represented by effective Hamiltonian with the Pauli matrices $\boldsymbol{\sigma}$ in terms of basis functions $|1\rangle$ and $|2\rangle$, calculated in second order of perturbation theory

$$
V_{Z}^{(3)}=\frac{3}{4} \Delta_{\mathrm{HL}} h^{3}\left(\sigma_{x} \cos 3 \varphi+\sigma_{y} \sin 3 \varphi\right),
$$

where dimensionless parameter $h=G_{\mathrm{h}} / \Delta_{\mathrm{HL}}$ is introduced. Equation (11) gives isotropic HH splitting $\omega_{Z}=(3 / 2) \Delta_{\mathrm{HL}} h^{3}$ and $\psi$-function phase $\theta=3 \varphi$. The polarization, $\rho_{\alpha}^{(0)}=-P_{\mathrm{eh}} \cos 2 \alpha$, is $\varphi$-independent. If $G_{\mathrm{e}}<T_{\mathrm{e}}$ and $h^{2} G_{\mathrm{h}}<T_{\mathrm{h}}$, one can find $P_{\mathrm{eh}} \approx G_{\mathrm{h}}^{3} G_{\mathrm{e}} / T_{\mathrm{e}} T_{\mathrm{h}} \Delta_{\mathrm{HL}}^{2} \propto B^{4}$. The $\mathrm{LH}$ admixture gives rise to the corrections $\delta \rho_{\alpha}^{(2)}$ which for important cases of $\alpha=0$ and $\alpha=45^{\circ}$ are $\delta \rho_{0}^{(2)}=-h^{2}$ and $\delta \rho_{45}^{(2)}=0$.

\subsection{Non-Zeeman interaction with a magnetic field}

Symmetry of Luttinger Hamiltonian admits the existence of non-Zeeman interaction of holes with a magnetic field [8], which has the form

$$
\boldsymbol{V}_{q}=q_{1} G_{\mathrm{h}}\left(J_{x}^{3} \cos \varphi+J_{y}^{3} \sin \varphi\right),
$$

where $q_{1}$ is a relatively small parameter reflecting the interaction between valence and $\Gamma_{15}$-conduction bands [9]. Let us note that in terms of the approach of [10], the real magnetic field $B$ substitutes $G_{\mathrm{h}}$ in (12). Thus, the Luttinger parameter $q$ appears in the form $q_{1}=q B / G_{\mathrm{h}}$. If the consideration of [10] would be supplemented by mechanisms sensitive to the exchange field influence, the latter equality could be modified. Presence of non-zero matrix elements of $V_{q}$ between HH states $|3 / 2\rangle$ and $|-3 / 2\rangle$ leads to the effective Hamiltonian in first order of perturbation

$$
V_{q}^{(1)}=\frac{3}{4} \Delta_{\mathrm{HL}} q_{1} h\left(\sigma_{x} \cos \varphi-\sigma_{y} \sin \varphi\right) .
$$

It gives isotropic HH splitting $\omega_{q}=(3 / 2) q_{1} G_{\mathrm{h}}$ and $\psi$-function phase $\theta=-\varphi$. The in-plane $g$-tensor $g_{\mu \nu}^{\perp}$ that can be defined in terms of Eq. (13) for HH pseudospin [9] $\widetilde{s}_{x}=\sigma_{x} / 2$ and $\widetilde{s}_{y}=\sigma_{y} / 2$ is isotropic, i.e. $g_{x x}^{\perp}=g_{y y}^{\perp}, g_{x y}^{\perp}=g_{y x}^{\perp}=0$. Nevertheless, it leads to a $\pi / 2$-periodical OPA, $\rho_{\alpha}^{(0)}=-P_{\text {eh }} \cos (4 \varphi+2 \alpha)$. It correlates with a cubic anisotropy of Luttinger Hamiltonian.

\subsection{Potentials of $C_{2 v}$ symmetry}

Two reasons for appearance of $C_{2 v}$ hole potential in QW are usually considered. The first is a $C_{2 v}$ constituent (so-called interface $C_{2 v}$ potential) of heterojunction potential inherent in [001] oriented structures [11, 12]. In structures with common anion (cation), the contributions of two QW interface potentials compensate each other. This compensation is not complete in the case of non-identical barriers or interface profile [3]. This interaction can be written in the form [11] $V_{i f}=$ $t_{i f}\left\{J_{x}, J_{y}\right\}$. Here $t_{i f}$ is an interaction constant and $\left\{J_{x}, J_{y}\right\} \equiv\left(J_{x} J_{y}+J_{y} J_{x}\right) / 2$.

Additionally to $V_{i f}$, there is also $C_{2 v}$ potential $V_{d}=d \varepsilon_{x_{d} y_{d}}\left\{J_{x_{d}}, J_{y_{d}}\right\}$ caused by in-plane strains [6]. Here $d$ is a deformation potential; $\varepsilon_{x_{d} y_{d}}$ is the strain with 


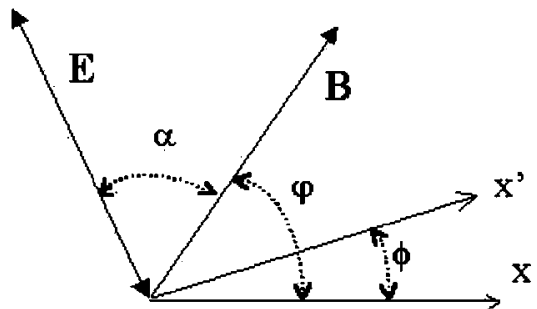

Fig. 1. The relative positions of the structure axis $x$, the axis $x^{\prime}$ of $C_{2 v}$ interaction (14), direction of in-plane magnetic field $\boldsymbol{B}$ and the line $E$ of intersection of the plane of polarization with the (001) plane.

$x_{d}$ and $y_{d}$ principal axes forming some angle with [100] and [010] axes. The sum of $V_{i f}$ and $V_{d}$ is also $C_{2 v}$ potential $V_{\mathrm{t}}$ with total amplitude $T_{\mathrm{t}}$ and axes $x^{\prime}$ and $y^{\prime}$ forming angle $\phi$ with [100] and [010] directions (see Fig. 1). Thus,

$$
V_{\mathrm{t}}=T_{\mathrm{t}}\left\{J_{x^{\prime}}, J_{y^{\prime}}\right\} \text {. }
$$

The potential (14) does not lift the $\pm 3 / 2 \mathrm{HH}$ degeneracy but results in $\mp 1 / 2$ LH admixture in first order of perturbation theory. This generates some temperature and magnetic field independent polarization [3] $\delta \rho_{\alpha}^{(1)}=-t \sin 2(\varphi-$ $\phi+\alpha)$, where $t=T_{\mathrm{t}} / \Delta_{\mathrm{HL}}$. The interference of $V_{Z}$ (10) and $V_{\mathrm{t}}$ (14) generates an effective in-plane $g$-factor for HHs [6]. It may be described by the Hamiltonian

$$
V_{\mathrm{ht}}^{(2)}=-\frac{3}{2} \Delta_{\mathrm{HL}} h t\left[\sigma_{x} \sin (\varphi+2 \phi)-\sigma_{y} \cos (\varphi+2 \phi)\right],
$$

which defines the phase $\theta=\varphi+2 \phi+\pi / 2$ and isotropic HH splitting $\omega_{\text {ht }}=$ $3 \Delta_{\mathrm{HL}} h t$. Nevertheless, the polarization, $\rho_{\alpha}^{(0)}=-P_{\mathrm{eh}} \sin 2(\varphi-\phi+\alpha)$, is anisotropic, $\pi$-periodical. If $G_{\mathrm{e}}<T_{\mathrm{e}}, \omega_{\mathrm{ht}}<T_{\mathrm{h}}$, one can find $P_{\mathrm{eh}} \approx 3 G_{\mathrm{h}} G_{\mathrm{e}} / 4 T_{\mathrm{e}} T_{\mathrm{h}} \propto B^{2}$ in agreement with data [2]. Equation (15) determines anisotropic HH $g$-tensor $g_{x^{\prime} x^{\prime}}^{\perp}=g_{y^{\prime} y^{\prime}}^{\perp}=0, g_{x^{\prime} y^{\prime}}^{\perp}=g_{y^{\prime} x^{\prime}}^{\perp}$. Let us note that in [2] there were used the reference system axes $x^{\prime \prime} \|[110]$ and $y^{\prime \prime}||\left[1 \overline{10}\right.$ ] i.e. rotated relative $x$ and $y$ by $45^{\circ}$ about the $z$-axis. It leads to: $g_{x^{\prime \prime} x^{\prime \prime}}^{\perp}=-g_{y^{\prime \prime} y^{\prime \prime}}^{\perp}, g_{x^{\prime \prime} y^{\prime \prime}}^{\perp}=g_{y^{\prime \prime} x^{\prime \prime}}^{\perp}=0$.

In-plane asymmetry of localized by a random potential (interface roughness, defects, etc.) hole $\psi$-function leads to mixing of $\mathrm{HH}$ and LH states [9], too. It can be represented as the sum of $C_{2 v}$ potentials (see [13] for details) with dispersed local main axes. If there is no preferential direction for $\mathrm{HH}$ localization in QW plane, this potential cannot lead to OPA. The numerical analysis [5] shows that random potential can suppress the OPA as soon as it exceeds other regular (non-random) interactions.

\section{Interference effects. Discussion}

The HH splitting was obtained isotropic for each HH Hamiltonian (11), (13), and (15). Let us consider joint manifestation of interactions that can split $\mathrm{HH}$ states 


$$
V=V_{Z}^{(3)}+V_{q}^{(1)}+V_{\mathrm{ht}}^{(2)}=\frac{1}{2}\left(\omega_{Z} \mathrm{e}^{-\mathrm{i} 3 \varphi}+\omega_{q} \mathrm{e}^{\mathrm{i} \varphi}+\omega_{\mathrm{ht}} \mathrm{e}^{-\mathrm{i}(\varphi+2 \phi+\pi / 2)}\right) .
$$

The HH splitting for this interaction can be written in terms of two expressions $Q$ and $R$ :

$$
\begin{aligned}
& \omega_{\mathrm{h}}=\frac{3}{2} \Delta_{\mathrm{HL}} h \sqrt{Q^{2}+R^{2}} \\
& Q=2 t \cos 2(\varphi-\phi)-q_{1} \sin 4 \varphi \\
& R=h^{2}+2 t \sin 2(\varphi-\phi)+q_{1} \cos 4 \varphi
\end{aligned}
$$

The polarization $\rho_{\alpha}$ for $\alpha=0$ and $\alpha=45^{\circ}$ can be now written as

$$
\rho_{\alpha}=\left\{\begin{array}{rrr}
-P_{\mathrm{eh}} \frac{R}{\sqrt{Q^{2}+R^{2}}}-h^{2}-t \sin 2(\varphi-\phi), & \alpha=0, \\
-P_{\mathrm{eh}} \frac{R}{\sqrt{Q^{2}+R^{2}}}-t \cos 2(\varphi-\phi), & \alpha=45^{\circ} .
\end{array}\right.
$$

Equation (20) with Eqs. (8) and (17) are the final results of our calculations that cover the most practically important cases.

One can see that HH splitting (17) reveals an anisotropy with finite magnitudes of $t$ and $q_{1}$ despite the isotropic character of HH splitting of each of term (16) taken separately. Moreover, the effective $H H$ transversal $g$-factor $\tilde{g}_{\perp}=\omega_{\mathrm{h}} / G_{\mathrm{h}}$ can be turned to zero at some direction and value of effective magnetic field (crossing of split HH sublevels). Simplest solution of the equation $\widetilde{g}_{\perp}=\frac{3}{2} \sqrt{Q^{2}+R^{2}}=0$ in the case of $\phi=0$ gives $\varphi= \pm \pi / 4$ and $h=\sqrt{ \pm 2 t+q_{1}}$.

As it is obvious from Eq. (20), the anisotropy and possible random degeneration of HH splitting do not influence OPA for high temperatures $T_{\mathrm{h}}>\omega_{\mathrm{h}}$, (or $P_{\mathrm{eh}} \propto \omega_{\mathrm{h}}$ ). In this case the different OPA contribution are additive and $\rho_{0}^{(0)} \approx-\frac{3}{2} R \frac{G_{\mathrm{h}}}{T_{\mathrm{h}}} \tanh \left(\omega_{\mathrm{e}} / 2 T_{\mathrm{e}}\right), \rho_{45}^{(0)} \approx-\frac{3}{2} Q \frac{G_{\mathrm{h}}}{T_{\mathrm{h}}} \tanh \left(\omega_{\mathrm{e}} / 2 T_{\mathrm{e}}\right)$, i.e. the $\pi$-periodical
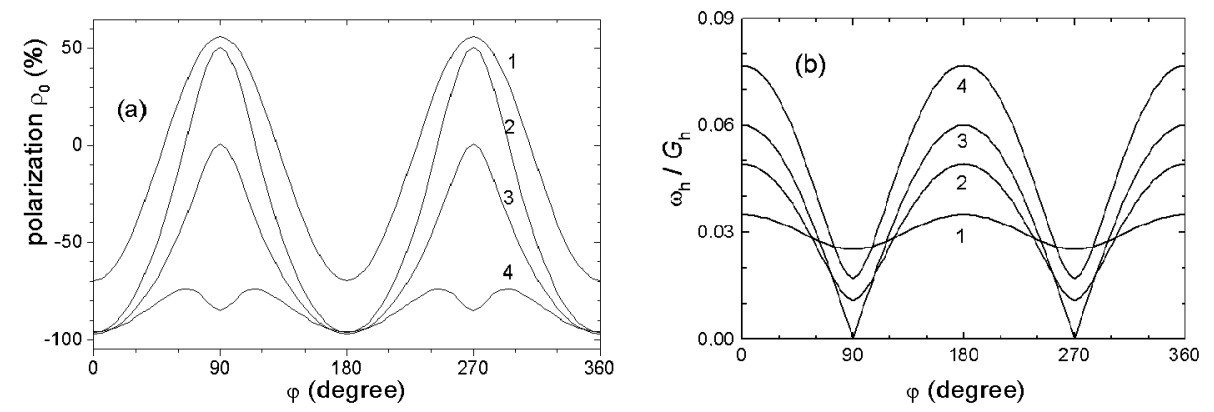

Fig. 2. The OPA (a) and transversal effective $H H g$-factor $\widetilde{g}_{\perp}=\omega_{\mathrm{h}} / G_{\mathrm{h}}$ (b) calculated for $q_{1}=0, t=0.01, \Delta_{\mathrm{HL}}=125 \mathrm{meV}, T_{\mathrm{e}}=T_{\mathrm{h}}=2 \mathrm{~K}$, and few magnitudes of magnetic field strength $h=-c \sqrt{2 t}$. The values $c 0.4,0.8,1$, and 1.25 are used for the curves $1-4$ correspondingly. 
OPA is proportional to $t$ and $\pi / 2$-periodical one is proportional to $q_{1}$. If $\mathrm{HH}$ splitting is not small as compared to temperature $T_{\mathrm{h}}$, Eq. (20) leads to qualitatively different character of OPA in the range of angles $\varphi$ corresponding to $\widetilde{g}_{\perp} \approx 0$ and $h \geq \sqrt{2|t|}$. Figure 2 reports some calculated curves of OPA and corresponding effective $g$-factor anisotropy.

Quantitative analysis carried out in terms of Eqs. (20) and (17) shows that experimental data of Ref. [1], where $\pi$ - and visible $\pi / 2$-periodical components of the OPA were detected from DMS-based QW, may be described nicely with appropriate set of parameters (see [5] for detail).

\section{Conclusion}

It is shown that two types of optical polarization contributions should be taken into account for describing OPA in QWs subjected to the in-plane magnetic field. The first of them arises owing to phase correlation of electron and hole wave functions, which is determined by a HH splitting in a magnetic field. The second one is due to admixture of $\mathrm{LH}$ to $\mathrm{HH}$ states.

The Zeeman interaction, $q$ term of the Luttinger Hamiltonian, and $C_{2 v}$ potentials are considered as sources of different OPA. Their joint manifestation reveals peculiar OPA behavior due to interference effects. Some new effects such as (i) the anisotropy of $\mathrm{HH}$ splitting due to interference of different $\mathrm{HH}$ potentials, (ii) polarization suppression at the conditions of crossing (anticrossing) of HH levels, (iii) depolarization effect of random potential influence are predicted. The nature of $\pi / 2$-periodical contribution to OPA caused by $q_{1}$ term is explained. The theory gives qualitative description for some important experimental details of OPA found earlier.

\section{Acknowledgment}

This paper was partly supported by grant INTAS 03-51-5266.

\section{References}

[1] O. Krebs, P. Voisin, Phys. Rev. Lett. 77, 1829 (1996).

[2] Yu.G. Kusrayev, A.V. Koudinov, I.G. Aksyanov, B.P. Zakharchenya, T. Wojtowicz, G. Karczewski, J. Kossut, Phys. Rev. Lett. 82, 3176 (1999).

[3] A. Kudelski, A. Golnik, J.A. Gaj, F.V. Kyrychenko, G. Karczewski, T. Wojtowicz, Yu.G. Semenov, O. Krebs, P. Voisin, Phys. Rev. B 64, 045312 (2001).

[4] S.M. Ryabchenko, O.V. Terletskij, Yu.G. Semenov, F.V. Kyrychenko, in: Optical Properties of 2D Systems with Interacting Electrons, Eds. W. Ossau, R. Suris, Kluwer Acad., Dordrecht 2003, p. 217.

[5] Yu.G. Semenov, S.M. Ryabchenko, Phys. Rev. B 68, 045322 (2003).

[6] G.E. Pikus, F.G. Pikus, Solid State Commun. 89, 319 (1994). 
[7] Diluted Magnetic Semiconductors, Eds. J.K. Furdyna, J. Kossut, Vol. 25 of Semiconductors and Semimetals, Academic, Boston 1988.

[8] G.L. Bir, G.E. Pikus, Symmetry and Strain Induced Effects in Semiconductors, Wiley, New York 1974.

[9] X. Marie, T. Amand, P. Le Jeune, M. Paillard, P. Renucci, L.E. Golub, V.D. Dymnikov, E.L. Ivchenko, Phys. Rev. B 60, 5811 (1999).

[10] C. Hensel, K. Suzuki, Phys. Rev. Lett. 22, 838 (1969).

[11] E.L. Ivchenko, A.A. Toropov, P. Voisin, Fiz. Tverd. Tela 40, 1925 (1998) [Phys. Solid State 40, 1748 (1998)].

[12] D.R. Yakovlev, A.V. Platonov, E.L. Ivchenko, V.P. Kochereshko, C. Sas, W. Ossau, L. Hansen, A. Waag, G. Landwehr, L.W. Molenkamp, Phys. Rev. Lett. 88, 257401 (2002).

[13] Yu.G. Semenov, K.N. Borysenko, K.W. Kim, Phys. Rev. B 66, 113302 (2002). 